POS PROCEEDINGS

\title{
KAON 2007: Conference Summary
}

\section{Gino Isidori*i}

INFN, Laboratori Nazionali di Frascati, Via E. Fermi 40, I-00044 Frascati, Italy

E-mail: gino.isidori@lnf.infn.it

A concise overview of the interesting recent developments in kaon phyiscs discussed at KAON 2007 is presented.

\section{KAON International Conference}

May 21-25 2007

Laboratori Nazionali di Frascati dell'INFN, Rome, Italy

\footnotetext{
*Speaker.

†This work is supported in part by by the EU Contract No. MRTN-CT-2006-035482, "FLAVIAnet".
} 


\section{Introduction}

There is no doubt that kaon physics has played a key role in our understanding of fundamental interactions in the last 60 years: several corner stones of the Standard Model (SM) have been derived, directly or indirectly, from $K$ meson phenomenology. What is less obvious, looking at this field from outside, is that kaon physics is still a very interesting subject. As I will try to summarise in the next five sections, the large number of interesting new results and stimulating discussions presented at this conference provide a clear demonstration of this fact.

\section{2. $V_{u s}$ and the semileptonic challenge to the SM}

The progress in the determination of $\left|V_{u s}\right|$ and, more generally, of the precise SM tests in $K_{\ell 2}$ and $K_{\ell 3}$ decays can be considered the main highlight of this conference. Before discussing the new experimental and theoretical results, it is worth spending few words about the interest of these measurements in general terms.

The determination of $\left|V_{u s}\right|$ has now reached the $0.5 \%$ level of accuracy [1]. Thanks to the smallness of $\left|V_{u b}\right|$ and the high-precision of $\left|V_{u d}\right|$, this implies we can test at per-mil level the relation

$$
\left|V_{u d}\right|^{2}+\left|V_{u s}\right|^{2}+\left|V_{u b}\right|^{2}=1 .
$$

Beside testing the unitarity of the CKM matrix, which is naturally satisfied in the SM and in most of its extensions, the main interest hidden behind Eq. (2.1) is a very stringent test of the universality of weak interactions. As reminded us by Marciano [2], when extracting the $\left|V_{i j}\right|$ from a given $u_{i} \rightarrow d_{j} \ell v$ process, the corresponding rate is normalised to $G_{F}^{(\mu)}$, or the Fermi coupling determined from the muon decay. As a result, testing the unitarity relation in Eq. (2.1) is equivalent to testing the universality of weak interactions between quarks and leptons.

Indeed, what is probed in low-energy experiment is not the gauge coupling of the $W$ to fermion fields (which, by construction, is insensitive to new physics) but effective four-fermion interactions of the type

$$
\mathscr{L}_{\text {eff. }}^{\text {C.C. }}=G_{F}^{(i j k l)} \bar{u}^{i} \Gamma d^{j} \bar{\ell}^{k} \Gamma v^{l}+\text { h.c. }
$$

The values of the effective couplings $G_{F}^{i j k l}$ are not protected by gauge invariance and are potentially sensitive to physics beyond the SM. On general grounds [3], one expects a power suppression of the non standard contributions of the type

$$
G_{F}^{(i j k l)}=\left[G_{F}^{(i j k l)}\right]_{\mathrm{SM}}\left[1+c \frac{M_{W}^{2}}{M_{\mathrm{NP}}^{2}}\right],
$$

where $M_{\mathrm{NP}}$ generically denotes the masses of the new particles. The coupling $c$ can be quite small if the new degrees of freedom appears only at the loop level $\left[c \sim 1 /\left(16 \pi^{2}\right)\right]$; but the high precision reached in a few semileptonic $K, \pi$ and nuclear $\beta$ decays can partially compensate such suppression. For instance, comparing $G_{F}^{(\mu)}$ with the effective coupling

$$
G_{F}^{(\mathrm{CKM})}=G_{F} \times\left[\left|V_{u d}\right|^{2}+\left|V_{u s}\right|^{2}\right],
$$

determined from $K_{\ell 3}$ and nuclear $\beta$ decays, allows us to exclude new heavy gauge bosons below $1 \mathrm{TeV}$ in specific beyond-SM frameworks [2]. 
A complementary class of weak universality tests is the comparison of the $\left|V_{u s}\right|$ values, or better the effective weak couplings, extracted from $K_{e 3}$ (vector coupling only), $K_{\mu 3}$ (vector and scalar coupling), and $K_{\ell 2}$ (scalar coupling only). Several beyond-SM frameworks are severely constrained by such tests. For instance, right-handed currents which are naturally present in Higgs-less models can induce $\mathscr{O}(1 \%)$ violations of universality between $K_{e 3}$ and $K_{\mu 3}$ [4]. Similarly, in two-Higgs doublet models with large $\tan \beta\left(\tan \beta=\left\langle H_{U}\right\rangle /\left\langle H_{D}\right\rangle\right)$ one expects violations of universality between $K_{\mu 2}$ and $K_{e 3}$ around few $\times 0.1 \%$ [5]. Precise measurements of the decay distributions in $K_{\ell 2}$ and $K_{\ell 3}$ decays could also constrain (or find evidences of) models with new light scalar particles, such as the $v$ MSM [6].

Last but not least, a particularly sensitive probe of new physics in semileptonic $K$ decays is the universality ratio

$$
R_{K}^{e / \mu}=\frac{\mathscr{B}(K \rightarrow e v)}{\mathscr{B}(K \rightarrow \mu v)} .
$$

This observable can be predicted to $0.04 \%$ accuracy within the SM [7], while its deviation from the SM can reach $\sim 1 \%$ in realistic supersymmetric frameworks with new sources of lepton-flavour mixing [5].

In summary, the measurements of $K_{\ell 2}$ and $K_{\ell 3}$ decay parameters have reached a level of precision which allows us to perform new significant tests of the SM. These precision tests are equally interesting and fully complementary to the flavour-conserving electroweak precisions tests and to the FCNC tests performed at $B$ factories.

\subsection{On the theoretical description of $K_{\ell 2}$ and $K_{\ell 3}$ decays}

The high statistics on semileptonic $K$ decays accumulated in the last few years have motivated a substantial progress in the theoretical description of these processes. In several observables the theoretical control is such that even the tiny deviations of lepton-flavor and/or quark-lepton universality mentioned above $(0.1 \%-1 \%)$ can possibly be detected.

The two master formulae for the inclusive decay rates are

$$
\begin{aligned}
& \Gamma\left(K_{\ell 3[\gamma]}\right)=C_{K_{\ell 3}}^{0} I^{K \ell}\left(\lambda_{i}\right) \times\left|V_{u s} f_{+}(0)\right|^{2} \times\left[1+2 \Delta_{S U(2)}^{K}+2 \Delta_{\mathrm{EM}}^{K \ell}\right], \\
& \Gamma\left(K_{\ell 2[\gamma]}\right)=C_{K_{\ell 2}}^{0} m_{\ell}^{2}\left(m_{K}^{2}-m_{\ell}^{2}\right) \times\left|V_{u s} f_{K}\right|^{2} \times\left[1+2 \Delta_{\mathrm{EM}}^{\ell}\right] .
\end{aligned}
$$

Here $C_{K}^{0}$ are overall coefficients which can be predicted with excellent accuracy (within the SM) in terms of $G_{F}^{(\mu)}$ and $\alpha_{\mathrm{em}}$, while the phase-space factors $I^{K \ell}$ can be determined by experiments measuring the Dalitz plot slopes. The CHPT calculations of isospin-breaking and radiative-correction factors ( $\Delta$ 's) have recently been completed, cross-checked and scrutinized against variations of the input parameters [7]. They are known with errors which range from a few $\times 10^{-4}$ to a few $\times 10^{-3}$, with the largest uncertainty coming from the isospin-breaking term

$$
\Delta_{S U(2)}^{K}=\left(\Delta_{S U(2)}^{K^{+}}-\Delta_{S U(2)}^{K^{0}}\right)=(2.3 \pm 0.2) \times 10^{-2} .
$$

The largest theoretical uncertainties (1.0-0.5\%) are still confined in the two overall hadronic form factors, $f_{K}$ and $f_{+}(0)$, whose best determinations are presently obtained from Lattice QCD. These uncertainties drop out in universality ratios, such as $R_{K}^{e / \mu}$ in Eq. (2.5). As a result, the 

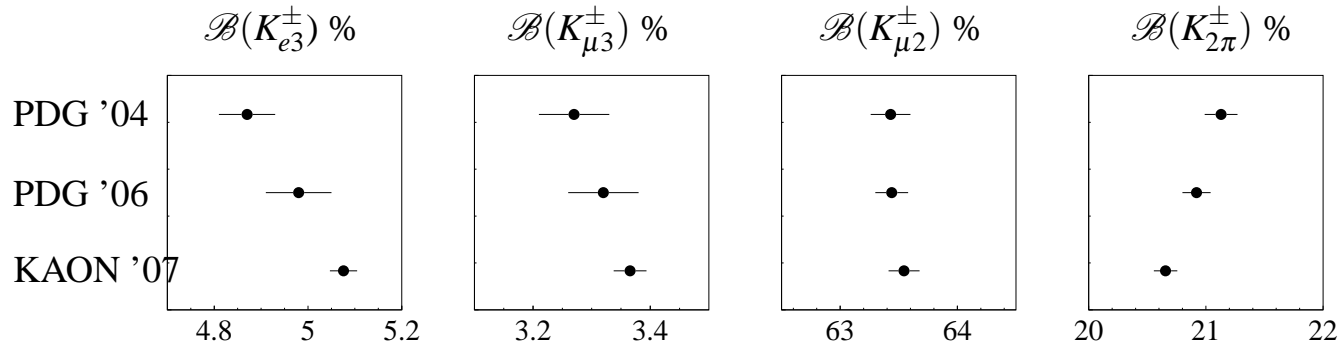

Figure 1: Evolution in the measurements of some of the leading $K^{ \pm}$decay rates [1].

precision in testing lepton-flavour universality or isospin-breaking effects is nowadays mainly an experimental issue. The limiting uncertainty in extracting $V_{u s}$ and in precision tests involving $f_{K}$ and $f_{+}(0)$ is still dominated by theory errors. However, the very recent progress of unquenched Lattice simulations (see sect. 2.3) allows to dream reaching $\mathscr{O}\left(10^{-3}\right)$ accuracies also in these cases in a near future.

\subsection{Experimental status of $K_{\ell 3}$ decays}

One of the virtues of the kaon system is that there is only a handful of leading decay modes, or branching ratios (BRs) above the $10^{-4}$ level. At fixed target experiments (KTeV, NA48 and ISTRA) the relative rates of these leading BRs are measured, while at KLOE, where the normalization of the kaon flux is known, direct measurements of the absolute branching ratios are also possible. The combination of all these data, together with life-time measurements, allows us to over-constrain the system. It is then possible to check the consistency of the various results and, possibly, to reduce the errors via global fits [8].

In the $K_{L}$ system there is a good overall agreement of the results produced by the new generation of kaon experiments since more than one year. The situation is indeed almost unchanged since the latest PDG fit [9]. On the other hand, a significant improvement has occurred at this conference in the charged kaon system, thanks to new results announced by KLOE (absolute $\mathscr{B}\left(K_{\ell 3}^{+}\right)[10]$ and $\left.\tau^{+}[11]\right)$, NA48 $\left(\mathscr{B}\left(K_{\ell 3}^{+}\right) / \mathscr{B}\left(K_{\pi \pi}^{+}\right)[12]\right)$ and ISTRA $\left(\mathscr{B}\left(K_{\ell 3}^{+} / \mathscr{B}\left(K_{\pi \pi}^{+}\right)\right.\right.$[13]). As shown in Fig. 1, there has been a substantial progress in the determination of the semileptonic branching ratios compared to the PDG 2006 values.

Beside the improvement in the final determination of $V_{u s}$, the most interesting aspect of the progress on $K^{ \pm}$semileptonic branching ratios is the direct (and precise) determination of isospinbreaking effects from data [1]:

$$
\left(\Delta_{S U(2)}^{K}\right)^{\exp }=(2.86 \pm 0.38) \times 10^{-2} .
$$

The good agreement of this result ${ }^{1}$ and the theoretical prediction in (2.8) is a great success of the CHPT calculations of isospin-breaking effects [14]. The consistency between theory and experiments in the leading isospin-breaking effects is reflected in the good consistency of the fit to

\footnotetext{
${ }^{1}$ This value is substantially different from the one quoted in Ref. [7] (based on the spring 2007 situation) mainly because of the new $\mathscr{B}\left(K_{\ell 3}^{ \pm}\right)$results by KLOE [10] and because of the modified $\mathscr{B}\left(K_{e 3}^{ \pm}\right)$value reported by NA48 [12].
} 


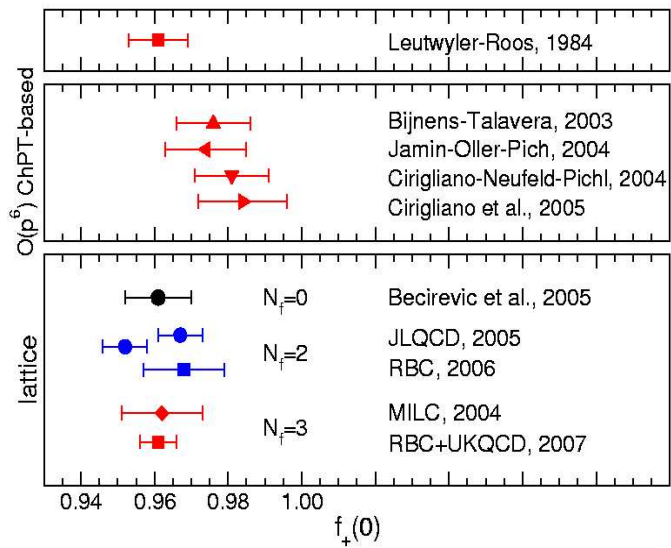

Figure 2: Comparison of analytic and lattice results for $f_{+}(0)$ [17] $\left(N_{f}\right.$ denote the number of dynamical fermions).

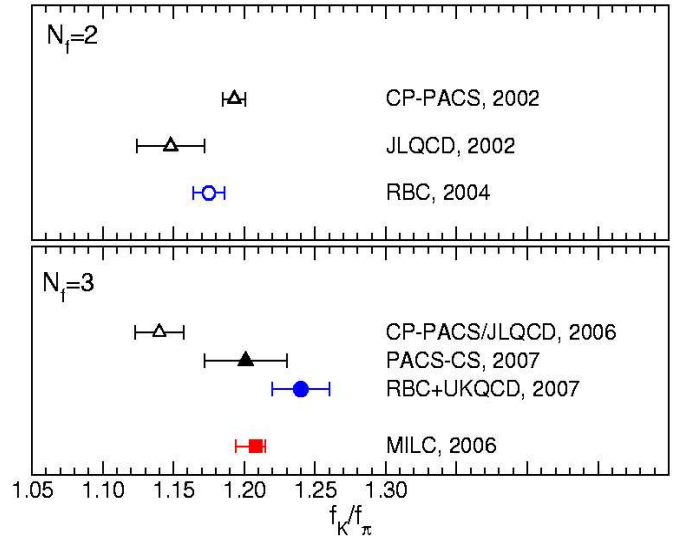

Figure 3: Comparison of recent unquenched and partially-quenched lattice results for $f_{K} / f_{\pi}$ [17].

$\left|V_{u s}\right| f_{+}(0)$ extracted from five different modes: $K_{e 3}^{L}, K_{\mu 3}^{L}, K_{e 3}^{S}, K_{e 3}^{ \pm}, K_{\mu 3}^{ \pm}$. The new global fit yields

$$
\left|V_{u s}\right| f_{+}(0)=0.21663(47)
$$

with $\chi^{2} / N_{\text {dof }}=2.62 / 4(\operatorname{Prob}=62 \%)$.

Despite the remarkable progress since 2004 (when CKM unitarity seemed to be violated at the $3 \sigma$ level...), it should stressed that we are still far from an ideal situation as far the experimental results are concerned. In the $K^{ \pm}$system not all the leading decay modes have been re-measured. More important, the recent measurements of slope and curvature of the form factors -especially the scalar slope- are not in good agreement [15]. This inconsistency has a minor direct impact in Eq. (2.10); however, it prevents us from performing interesting chiral tests (see Ref. [4, 16]) of the methods used to estimate $f_{+}(0)$ (including Lattice QCD). There is certainly room for significant improvements in view of KAON 2009!

\subsection{Lattice progress on $f_{+}(0)$ and $f_{K} / f_{\pi}$ and the extraction of $V_{u s}$}

As nicely summarised by Kaneko [17] and Juttner [18], in the last two years there has been a dramatic improvement in the quality of lattice simulations relevant to kaon form factors. The two main obstacles which so far prevented reliable estimates, namely the quenched approximation and the chiral extrapolation, have essentially been removed. The most recent simulations are performed with 2 or 3 dynamical fermions and with pion masses around or below $\approx 300 \mathrm{MeV}$, where the chiral extrapolation does not represent a severe problem.

The recent results for $f_{+}(0)$ and $f_{K} / f_{\pi}$ are shown in Fig. 2 and 3. In the case of $f_{+}(0)$ there is still some competition from analytical approaches, while in the case of $f_{K} / f_{\pi}$ only lattice results are able to reach the $\mathscr{O}(1 \%)$ level. This of course does not mean that analytical calculations of kaon form factors are useless. The non-trivial effort of computing these form factors in CHPT at one or two-loop level [16] is an essential tool to improve and control the chiral extrapolation of the recent lattice results. 


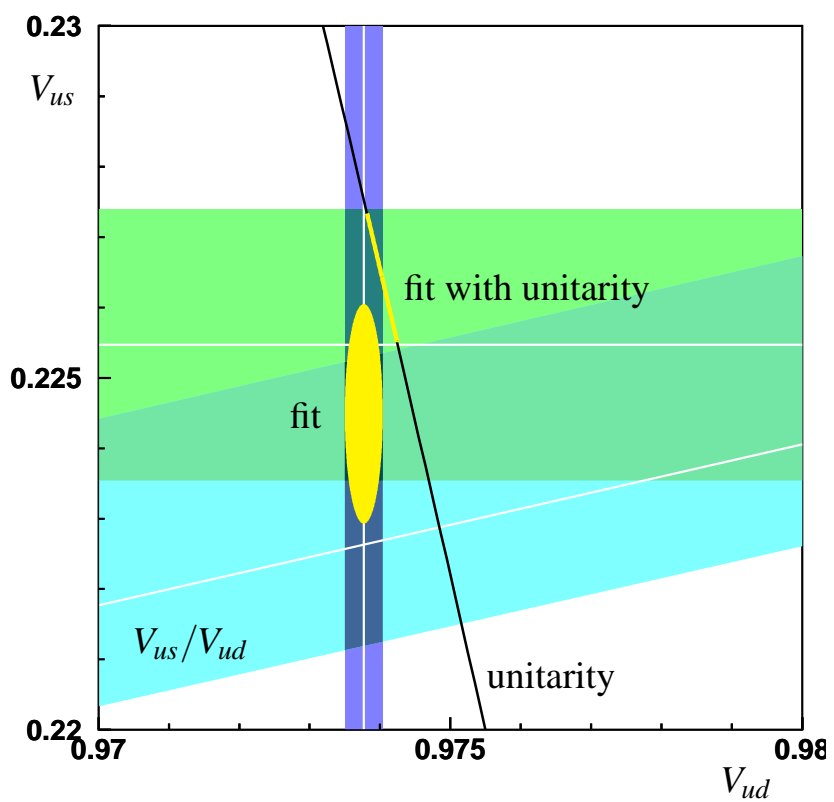

Figure 4: Results of the recent fits to $\left|V_{u s}\right|$ (from $K_{\ell 3}$ ) and $\left|V_{u s}\right| /\left|V_{u d}\right|$ (from $K_{\ell 2}$ ) compared to the world average value of $\left|V_{u d}\right|$.

As far as $f_{+}(0)$ is concerned, it is interesting to note that all lattice results, and especially the most recent and precise one by Ref. [20], are in excellent agreement with the old result by Leutwyler and Roos. At first sight this is somehow surprising given the large $\mathscr{O}\left(p^{6}\right)$ contributions appearing in CHPT at the two-loop level [19] (which are responsible for the large central values of $f_{+}(0)$ obtained in CHPT + large $N_{c}$ approaches [21]). On the other hand, it should be stressed that the $\mathscr{O}\left(p^{6}\right)$ CHPT calculation of this quantity is not complete, given the absence of a modelindependent information on the corresponding counterterms. If confirmed by a more reliable chiral extrapolation, the result of Ref. [20] would then provide a very useful insight about CHPT beyond the one-loop level.

The present global fit to $\left|V_{u d}\right|$ and $\left|V_{u s}\right|$ using the Leutwyler-Roos result for $f_{+}(0)$ and $f_{K} / f_{\pi}=$ $1.208(2)\left(_{-14}^{+7}\right)[22]$-used to determine $\left|V_{u s}\right| /\left|V_{u d}\right|$ from $\Gamma\left(K_{\ell 2}\right) / \Gamma\left(\pi_{\ell 2}\right)$ - is shown in Fig. 4. As can be noted, the agreement with CKM unitarity is excellent.

All the recent lattice results on kaon form factors at the $\mathscr{O}(1 \%)$ level are still based on a limited set of quark masses (especially in the case of $f_{+}(0)$ ) and/or specific actions (especially in the case $f_{K} / f_{\pi}$ ). Therefore the quoted errors should still be taken with some care. Nonetheless it is clear that we are entering a new era, where lattice will help us both to test the SM at the per-mil level and to shed light on the convergence of the chiral expansion beyond the one-loop level.

\subsection{The $R_{K}^{e / \mu}$ ratio}

As anticipated, the ratio in Eq. (2.5) can be computed to an excellent accuracy within the SM and provides an interesting probe of non-standard scenarios which violate lepton flavour universal- 


\begin{tabular}{lc}
\hline \hline & $R_{K}^{e / \mu}\left[10^{-5}\right]$ \\
\hline PDG 2006 [9] & $2.45 \pm 0.11$ \\
NA48/2 '03 prel. [25] & $2.416 \pm 0.043 \pm 0.024$ \\
NA48/2 '04 prel. [26] & $2.455 \pm 0.045 \pm 0.041$ \\
KLOE prel. [27] & $2.55 \pm 0.05 \pm 0.05$ \\
\hline SM prediction & $2.472 \pm 0.001$ \\
\hline \hline
\end{tabular}

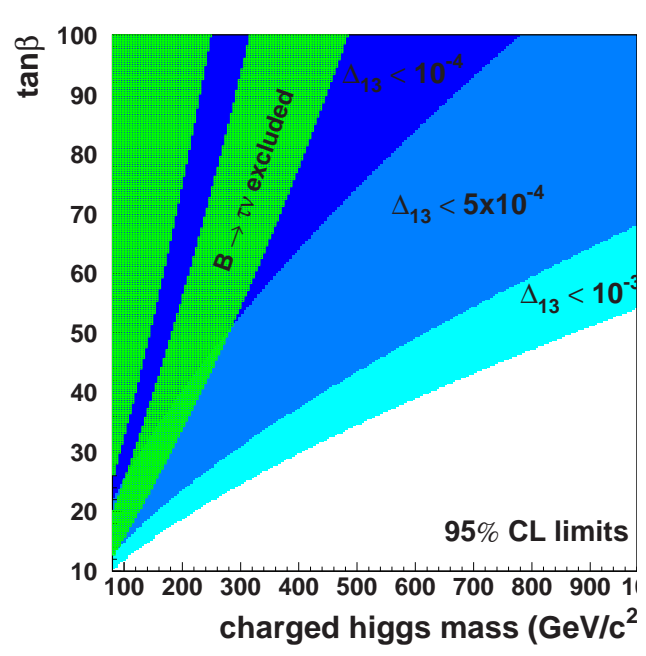

Figure 5: Current experimental data on $R_{K}^{e / \mu}$ (left). Exclusion limits at $95 \% \mathrm{CL}$ in the $\tan \beta-M_{H^{ \pm}}$plane from $R_{K}^{e / \mu}$ for different values of the LFV coupling $\Delta_{13}$ [23]. For comparison, the present limits from $B \rightarrow \tau \nu_{\tau}$ decays are also shown.

ity. The recent work of Ref. [23], which has pointed out the possibility of deviations from the SM of up to $\sim 1 \%$ in realistic supersymmetric scenarios, has triggered a renewed interest in the precise measurements of this ratio [24].

The limiting factor in the determination of $R_{K}^{e / \mu}$ is the $K \rightarrow e v$ rate, whose experimental knowledge has been quite poor so far. The current official world average, which implies $R_{K}^{e / \mu}=$ $(2.45 \pm 0.11) \times 10^{-5}$ dates back to experiments from the 70's [9]. The situation has finally changed thanks to a series of preliminary results by NA48/2 and KLOE (see Fig. 5), two of which have been announced at this conference. The two results by NA48/2, being based on different data sets (2003 and 2004, respectively) with different running conditions, should be regarded as completely independent. Combining these new results with the PDG value yields [24]

$$
R_{K}^{e / \mu}=(2.457 \pm 0.032) \times 10^{-5} .
$$

This result is in good agreement with the SM expectation and has a relative error $(\sim 1.3 \%)$ three times smaller compared to the previous world average.

In the supersymmetric framework of Ref. [23] this result implies significant constraints in the $\tan \beta-M_{H^{ \pm}}$plane (see Fig. 5). The constraints depend on the lepton flavour violating (LFV) coupling $\Delta_{13}$, whose natural values lies below $10^{-3}\left(\Delta_{13} \sim \delta_{R R}^{\ell} \times \alpha /(2 \pi)\right)$. It is interesting to note that even for $\Delta_{13} \sim 10^{-4}$ the bounds extracted from $R_{K}^{e / \mu}$ are competitive with the LFV-independent bounds presently derived from $B \rightarrow \tau \nu_{\tau}$.

Further improvements in the knowledge of $R_{K}^{e / \mu}$ are foreseen in the near future. The preliminary KLOE measurement has a conservative estimate of the systematic uncertainty and is not based on the full statistics. Adding the remaining statistics and improving the reconstruction efficiency should reduce its error down to the $1 \%$ level. An error on $R_{K}^{e / \mu}$ of about $0.3 \%$ is the ambitious goal of the 2007 dedicated run of the CERN-P326 collaboration (the successor of NA48) [26, 28]. If these expectations will be fulfilled, in a short term the world average of $R_{K}^{e / \mu}$ will decrease by an 
additional factor of four. This could open the possibility of finding clear deviations from the SM, or to set very stringent limits in the parameter space of realistic supersymmetric models.

\section{The mystic world of $\pi \pi$ phase shifts}

While $K_{\ell 2}$ and $K_{\ell 3}$ decays represent a unique observatory on electroweak interactions, $K_{3 \pi}$ and $K_{\ell 4}$ decays provide a privileged observatory on strong interactions. The key observables here are the $\pi \pi$ phase shifts near threshold, and particularly the two $S$-wave $\pi \pi$ scattering lengths. These hadronic observables can be predicted with an excellent accuracy in CHPT [29]

$$
\begin{aligned}
a_{0} m_{\pi^{+}} & =0.220 \pm 0.005, \quad a_{2} m_{\pi^{+}}=-0.044 \pm 0.001 \\
\left(a_{0}-a_{2}\right) m_{\pi^{+}} & =0.265 \pm 0.004 .
\end{aligned}
$$

The above results are direct consequences of the basic CHPT assumptions about the breaking of chiral symmetry in the QCD vacuum. Confronting these predictions with experiments is therefore a way to test and improve our knowledge about the QCD vacuum [30].

Till very recently the experiments where unable to match the high precision of the predictions in Eq. (3.1). The situation has substantially improved mainly thanks to the high-precision measurements of $K_{3 \pi}^{+}$and $K_{\ell 4}^{+}$decay distributions performed by NA48/2. However, the situation is far from being completely understood. The high accuracy of the new experimental results poses new challenging questions to the theoreticians [31]: how to relate the predictions in Eq. (3.1), which have been obtained in an ideal framework of perfect isospin symmetry (the paradise), to what is measured in experiments (the real world), where we cannot switch-off the isospin-violating electromagnetic interactions.

\subsection{The $K \rightarrow 3 \pi$ cusp}

As pointed out by Cabibbo in 2004 [32], the rescattering of the final state pions produces a prominent cusp in the $M_{\pi^{0}} \pi^{0}$ spectrum of the $K^{+} \rightarrow \pi^{+} \pi^{0} \pi^{0}$ decay. This effect can be used to obtain a precise determination of the $a_{I}$ and, particularly, of the $a_{0}-a_{2}$ combination. The NA48/2 collaboration has just released [33] a combined fit of their 2003+2004 data set using the parameterization of Ref. [34] (see Fig. 6).

The approach of Ref. [34] is based on a systematic expansion of the singular terms of the $M_{\pi^{0}} \pi^{0}$ distribution in powers of $\pi \pi$ scattering lengths: a framework which is more efficient (as far as the extraction of the $a_{I}$ is concerned) and substantially simpler than ordinary CHPT. However, this method allows us to take into account only the isospin-breaking effects which can be re-absorbed into the definition of pion masses and scattering lengths (what we can call factorisable radiative corrections). As can be noted, the agreement between data and theory is good but for a narrow region around the cusp, where the formation of the pionium occurs. Cutting out this region, the results of the fit are [33]

$$
\begin{aligned}
\left(a_{0}-a_{2}\right) & =0.261 \pm 0.006_{\text {stat. }} \pm 0.003_{\text {syst. }} \pm 0.001_{\text {ext. }} \\
a_{2} & =-0.037 \pm 0.013_{\text {stat. }} \pm 0.009_{\text {syst. }} \pm 0.002_{\text {ext. }}
\end{aligned}
$$



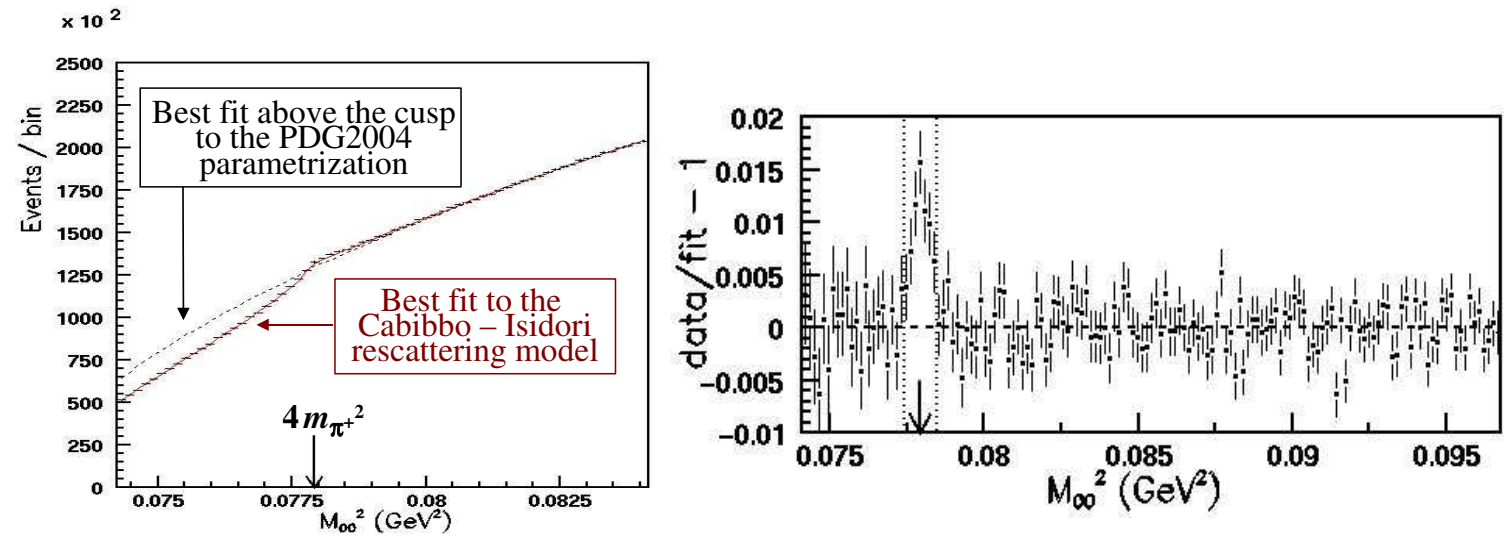

Figure 6: $M_{\pi^{0} \pi^{0}}$ spectrum in $K^{+} \rightarrow \pi^{+} \pi^{0} \pi^{0}$, close to the cusp region (left), compared to the PDG parameterization (no singularity) and the model of Ref. [34]. Deviation of the fitted spectrum (using the parameterization of Ref. [34]) and data (pulls) with statistical errors (NA48/2 combined 2003+2004 data set) [33].

where the theoretical error induced by the parameterization of Ref. [34] (estimated to be around $5 \%$ ) is not included. The small statistical error, the nice agreement with the theory predictions in Eq. (3.1), and the high quality of the global fit to the $M_{\pi^{0} \pi^{0}}$ distribution is quite impressive!

To fully exploit the high potential of this method (and these excellent data) a further improvement to reduce the theory error is needed. The recent works in Ref. [35, 36] are useful steps forward in this direction: they both confirm (at least numerically) the parameterization of Ref. [34] around the cusp in absence of non-factorisable electromagnetic corrections. Moreover, the tiny numerical difference of the three methods suggest that the $\mathscr{O}\left(a_{I}^{3}\right)$ terms are negligible. However, the key issue, namely the estimate of the non-factorisable radiative corrections, has not been addressed yet. The approach of the Bern group [35], based on a non-relativistic field theory, provides a systematic tool to evaluate these effects.

Last but not least, it is worth to stress that a further reduction of both theory and experimental errors on the $a_{I}$ extracted from this method requires a precise re-measurement of the $K^{+} \rightarrow \pi^{+} \pi^{+} \pi^{-}$channel [31]. Indeed the leading singularity in the $M_{\pi^{0} \pi^{0}}$ distribution arises by the $\pi^{+} \pi^{-} \rightarrow \pi^{0} \pi^{0}$ scattering, whose strength (compared to the regular terms) is controlled by the ratio $\Gamma\left(K^{+} \rightarrow \pi^{+} \pi^{+} \pi^{-}\right) / \Gamma\left(K^{+} \rightarrow \pi^{+} \pi^{0} \pi^{0}\right)$.

\section{$3.2 K_{\ell 4}$ decays and global fit to the $a_{I}$}

Historically $K_{\ell 4}$ decays have been the main source of information on $\pi \pi$ phase shifts. Here the four-body kinematics allows different isospin and angular-momentum states for the two-pions, which can be disentangled studying their angular distribution. Contrary to the cusp effect just discussed, the phase shifts are measured above threshold and the extraction of the scattering lengths is performed with the $s_{\pi \pi} \rightarrow 4 m_{\pi}^{2}$ extrapolation.

The first high-statistics analysis of the scattering lengths in $K_{\ell 4}$ has been performed a few years ago by the BNL-E865 [37] collaboration. Their results are in good agreement with the CHPT predictions in Eq. (3.1), although the final error is still quite large $\left(\approx 6 \%\right.$ on $a_{0}$, fixing the $a_{2}-$ 


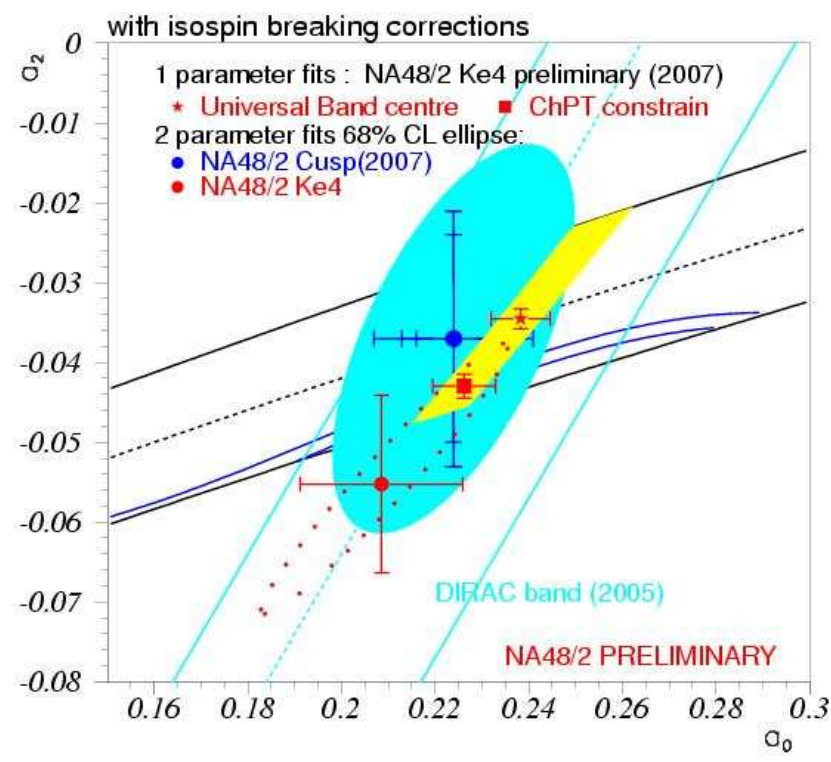

Figure 7: Recent NA48/2 results on the $a_{0}$ and $a_{2}$ [33].

$a_{0}$ correlation from theory). NA48/2 has just released a preliminary analysis of their 2003 data set which has already twice the statistic of the BNL-E85 experiment [38], and their full data set $(2003+2004)$ could allow to further double it. A final value for the $a_{I}$ has not been presented yet, but some interesting conclusions can already be drawn. Isospin-breaking effects in the phase shifts, which have been ignored in previous analyses, turn out to be quite relevant. Only after including these effects (which are still under theoretical investigation by Gasser and collaborators [31]), the $a_{I}$ extracted form $K_{\ell 4}$ are consistent with those derived from the cusp analysis (and with the theoretical predictions). The $a_{0}$ and $a_{2}$ values extracted from the $K_{\ell 4}$ analysis have a mild correlation, different from the one arising from the cusp effect (see Fig. 7). Imposing external theoretical constrains on the $a_{0}-a_{2}$ plane, as for instance done in Ref. [37], leads to sizable variations in the output results. On the other hand, the different correlation between cusp and $K_{\ell 4}$ data implies that combining these two data set we could finally challenge the theoretical predictions for the $a_{I}$ in a model-independent way.

\section{The chaotic world of non-leptonic and radiative decays}

The theoretical description of branching ratios and $\mathrm{CP}$ violating asymmetries in non-leptonic $K$ decays is very different from the pristine world of semileptonic $K$ decays and $\pi \pi$ scattering. Because of the complicated interplay of strong and weak interactions, theory is still well behind the high-precision level reached by experiments.

As discussed by C. Sachrajda and R. Mawhinney [39], in the long run there is a realistic hope that lattice QCD calculations of non-leptonic channels will get close to the precision reached by experiments. All conceptual problems involved in a full QCD calculation of $K \rightarrow 2 \pi$ amplitudes (in particular the delicate issue of final-state interactions) have been solved. What remain to be faced are practical problems: the $K \rightarrow 2 \pi$ amplitude requires the evaluation of 4-point Green functions 

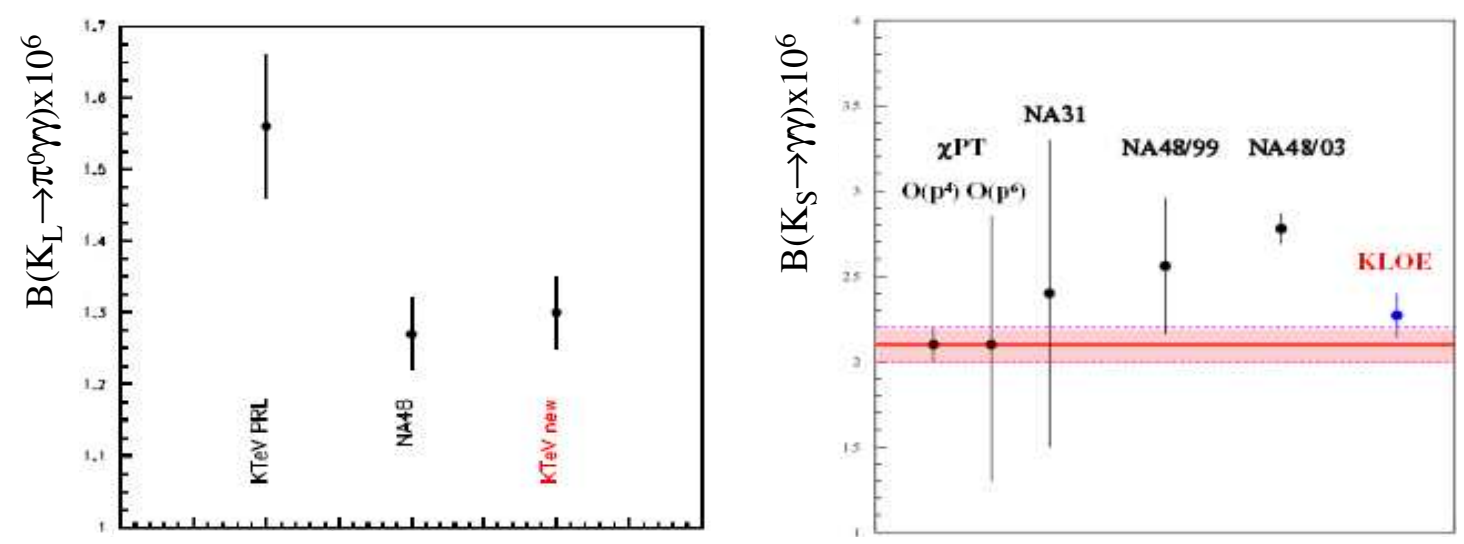

Figure 8: Recent results on two-photon modes: $\mathscr{B}\left(K_{L} \rightarrow \pi^{0} \gamma \gamma\right)$ from $\mathrm{KTeV}[45]$ and $\mathscr{B}\left(K_{S} \rightarrow \gamma \gamma\right)$ from KLOE [46].

of several operators (compared to the 2- and 3-point functions of a single operator involved in semileptonic form factors); an excellent control of chiral corrections is needed, and the control of final-state interactions require large volumes. At present, all this make impossible to directly confront theory and experiments in $K \rightarrow 2 \pi$, especially in delicate quantities such as $\varepsilon^{\prime} / \varepsilon$. But the situation is likely to improve in the future with more powerful CPU and improved algorithms.

In the meanwhile, experiments are producing new interesting results. The NA48/2 collaboration has announced the final results on the $K^{ \pm} \rightarrow(3 \pi)^{ \pm}$Dalitz-plot asymmetries [40]:

$$
\begin{aligned}
A_{g}^{c} & =\frac{g^{+}-g^{-}}{g^{+}+g^{-}}=(-1.5 \pm 2.1) \times 10^{-4} \quad\left[K^{ \pm} \rightarrow \pi^{ \pm} \pi^{+} \pi^{-}\right], \\
A_{g}^{n} & =(1.8 \pm 1.8) \times 10^{-4} \quad\left[K^{ \pm} \rightarrow \pi^{ \pm} \pi^{0} \pi^{0}\right],
\end{aligned}
$$

which show no evidence of CP violation at the $10^{-4}$ level. This result, which is expected within the SM by general arguments [41] (and confirmed by a detailed CHPT analysis [42, 43]), allows to exclude some exotic NP scenarios [44].

The situation of radiative non-leptonic $K$ decays is somewhat better than the pure non-leptonic sector since CHPT allows us to derive non-trivial relations [42]. We cannot test the electroweak sector of the SM, but we can understand better the interplay of strong and weak interactions at low energies. Particularly interesting are the two-photon modes $K_{S} \rightarrow \gamma \gamma$ and $K_{L} \rightarrow \pi^{0} \gamma \gamma$. The rates of these channels are unambiguously predicted in CHPT at $\mathscr{O}\left(p^{4}\right)$ and their precise measurements allow us to explore the largely unknown sector of CHPT at $\mathscr{O}\left(p^{6}\right)$. Here we have seen two interesting new experimental results (see Fig. 8). A new measurement of $\mathscr{B}\left(K_{L} \rightarrow \pi^{0} \gamma \gamma\right)$ by KTeV [45] has finally clarified the long-standing discrepancy with NA48 (incidentally, this is also a very good news for $K_{L} \rightarrow \pi^{0} e^{+} e^{+}$, since it implies that its CPC component is totally negligible. [47]). On the other hand, KLOE has announced a new measurement of the $K_{S} \rightarrow \gamma \gamma$ rate [46] which is barely consistent with a previous NA48 result. A clarification of the $K_{S} \rightarrow \gamma \gamma$ result would be useful to estimate the real size of the $\mathscr{O}\left(p^{6}\right)$ terms in channels which are free from vector-meson enhanced contributions. 


\section{Challenging the basis: CPT and QM tests}

The neutral kaon system offers a unique possibility to challenge some of the foundations of our description of fundamental interactions, such as CPT invariance and Quantum Coherence. Exact CPT invariance is expected in any quantum field theory respecting the general hypotheses of Lorentz invariance, locality and unitarity. However, these hypotheses are likely to be violated at very high energy scales, where the quantum effects of gravitational interactions cannot be ignored. As shown by Mavromatos [48], CPT non-invariance naturally arises in quantum-gravity frameworks as a consequences of Lorentz violations and, in specific frameworks, the CPT symmetry could even be ill-defined. On the phenomenological side, since we still miss a consistent theory of quantum gravity, it is hard to predict where and at which level CPT-violating effects may show up: this is an experimentally-driven search, where physical systems with the highest sensitivity, such as the neutral kaon system, play the leading role.

One of the most significant tests of CPT invariance is the one obtained by means of the BellSteinberger (BS) relation: a relation which makes use of unitarity (or the conservation of probability) to connect a violation of CPT in the neutral kaon Hamiltonian $\left(m_{K^{0}} \neq m_{\bar{K}^{0}}\right.$ and/or $\left.\Gamma_{K^{0}} \neq \Gamma_{\bar{K}^{0}}\right)$ to the observable CP-violating interference of $K_{L}$ and $K_{S}$ decays into the same final state $f$. The key equation following from the BS relation is [49]:

$$
\left[\frac{\Gamma_{S}+\Gamma_{L}}{\Gamma_{S}-\Gamma_{L}}+i \tan \phi_{\mathrm{SW}}\right]\left[\frac{\Re(\varepsilon)}{1+|\varepsilon|^{2}}-i \mathfrak{I}(\delta)\right]=\frac{1}{\Gamma_{S}-\Gamma_{L}} \sum_{f} \mathscr{A}_{L}(f) \mathscr{A}_{S}^{*}(f)
$$

where the two output parameters, $\varepsilon$ (CP violating and CPT conserving) and $\delta$ (CPT violating) are defined by

$$
\varepsilon \pm \delta=\frac{-i \mathfrak{I}\left(m_{12}\right)-\frac{1}{2} \mathfrak{I}\left(\Gamma_{12}\right) \pm \frac{1}{2}\left[m_{\bar{K}^{0}}-m_{K^{0}}-\frac{i}{2}\left(\Gamma_{\bar{K}^{0}}-\Gamma_{K^{0}}\right)\right]}{m_{L}-m_{S}+i\left(\Gamma_{S}-\Gamma_{L}\right) / 2} .
$$

The advantage of the neutral kaon system is that only few decay modes give a significant contribution to the r.h.s. of Eq. (5.1), which can be determined from experiments with good accuracy.

The results of a new global fit, including several new measurements by KLOE [50], are shown in Fig. 9. The error on $\mathfrak{I}(\boldsymbol{\delta})$ and the corresponding bound on CPT violating effects have been improved by about a factor of two compared to the PDG [9]. As stressed by several authors, the fact that the difference between $K^{0}$ and $\bar{K}^{0}$ masses is found to be smaller than few $\times 10^{-18} \mathrm{GeV}$ (see Fig. 9) could suggest that we are really probing Planck-scale physics. This fact should not be over-emphasized (there is no guarantee that CPT-violating effects scale linearly with $M_{K} / M_{\text {Planck }}$ ), but it is a clear demonstration of the unique sensitivity of the neutral kaon system.

The KLOE experiment is an ideal framework to make many other tests of CPT invariance and quantum coherence [50]. The most remarkable example is the limit set on the $K^{0}-\bar{K}^{0}$ decoherence parameter, $\zeta_{00}$, which can be bounded by the absence of equal-time and equal-final-state events in the $\Phi \rightarrow K^{0} \bar{K}^{0} \rightarrow f_{1}\left(t_{1}\right) f_{2}\left(t_{2}\right)$ decay chain. The recent KLOE analysis has set the bound $\zeta_{0 \overline{0}}<$ $5 \times 10^{-6}$ at $95 \%$ C.L. [51]. This result is five order of magnitude better (!) with respect to the previous limit on $\zeta_{0 \overline{0}}$ (set by CPLEAR) and with respect to limits recently set at $B$ factories ${ }^{2}$ on the corresponding $B$-physics observable [50].

\footnotetext{
${ }^{2}$ The great advantage of a $\Phi$ factory in this type of measurement is due to the smallness of $C P$ violation in the neutral kaon system, which strongly suppress the SM background.
} 


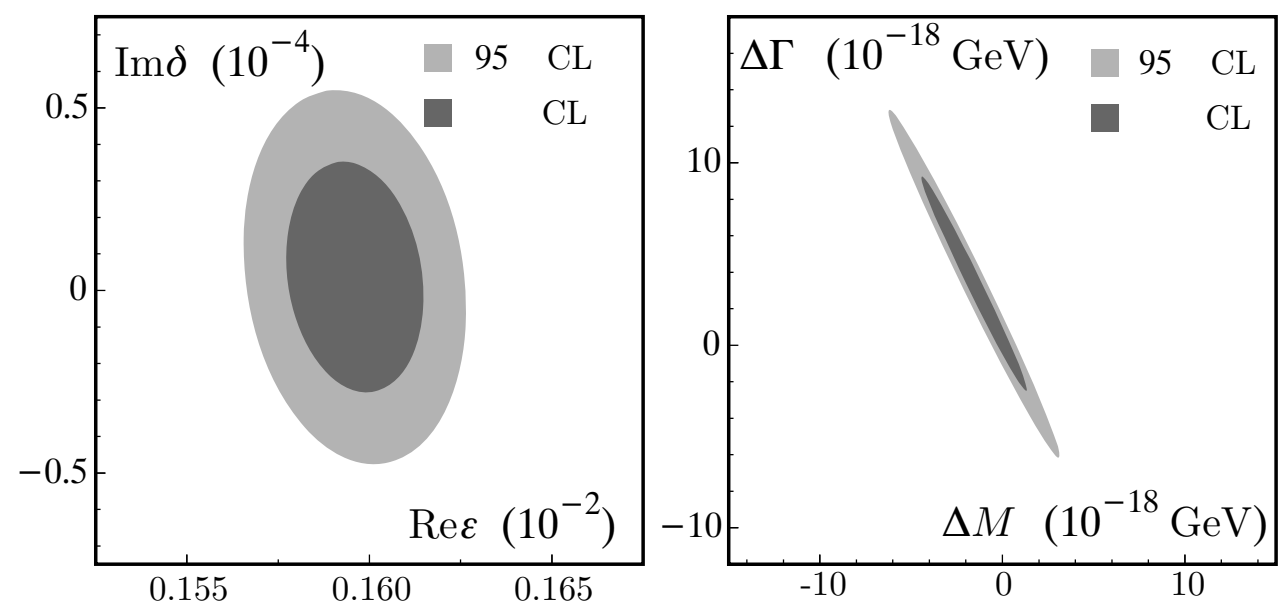

Figure 9: Results of the present global fit to the Bell-Steinberger relation [49]: allowed regions at $68 \%$ and 95\% C.L. in the $\mathfrak{R}(\varepsilon)-\mathfrak{I}(\boldsymbol{\delta})$ plane (left) and in the $\left(m_{K^{0}}-m_{\bar{K}^{0}}\right)-\left(\Gamma_{K^{0}}-\Gamma_{\bar{K}^{0}}\right)$ plane (right).

\section{The holy grail of kaon physics: $K \rightarrow \pi v \bar{v} \&$ other ultra-rare decays}

Rare decays mediated by flavour-changing neutral currents (FCNCs) are one of the best tools to investigate the flavour structure of physics beyond the SM. Among the many rare $K$ and $B$ decays, the $K^{+} \rightarrow \pi^{+} v \bar{v}$ and $K_{L} \rightarrow \pi^{0} v \bar{v}$ modes are unique since their SM branching ratios can be computed to an exceptionally high degree of precision, not matched by any other FCNC processes involving quarks. It is then not surprising that $K \rightarrow \pi \nu \bar{v}$ decays continue to raise a strong theoretical interest, both within and beyond the SM [52, 53, 54].

Beside several NP analyses [53,54], which confirm the high discovery potential of these channels (see Fig. 10), it is worth stressing the three important improvements on the SM predictions of $K \rightarrow \pi v \bar{v}$ rates since KAON 2005: i) the NNLO calculation of the dimension-six charm-quark contribution to $K^{+} \rightarrow \pi^{+} v \bar{v}$ [55]; ii) the first complete analysis of dimension-eight and longdistance (up-quark) contributions relevant to $K^{+} \rightarrow \pi^{+} v \bar{v}$ [56]; iii) a new comprehensive analysis of matrix-elements and isospin-breaking effects, relevant to both channels [57]. Thanks to these recent works, the irreducible theoretical uncertainties on both branching ratios are at the few $\%$ level. The present SM predictions are [52]

$$
\begin{aligned}
\mathscr{B}\left(K^{+} \rightarrow \pi^{+} v \bar{v}\right)_{\mathrm{SM}} & =(2.54 \pm 0.35) \times 10^{-11}, \\
\mathscr{B}\left(K_{L} \rightarrow \pi^{0} v \bar{v}\right)_{\mathrm{SM}} & =(7.96 \pm 0.86) \times 10^{-11},
\end{aligned}
$$

where a good fraction of the error (especially in the $K_{L}$ case) is the parametric uncertainty induced by $V_{t s}$ and $V_{t d}$, whose knowledge will certainly improve in the near future.

The slow experimental progress on these channels is a big shame of the particle-physics community (and, especially, of its funding system): several interesting proposal (even successfully running experiments) have been stopped or suspended for reasons which have nothing to do with physics. Fortunately this has not discouraged everybody! The P326 experiment at CERN [28] and the possibility to move and improve the E391a experiment [59] at the JPARC accelerator [60] are 


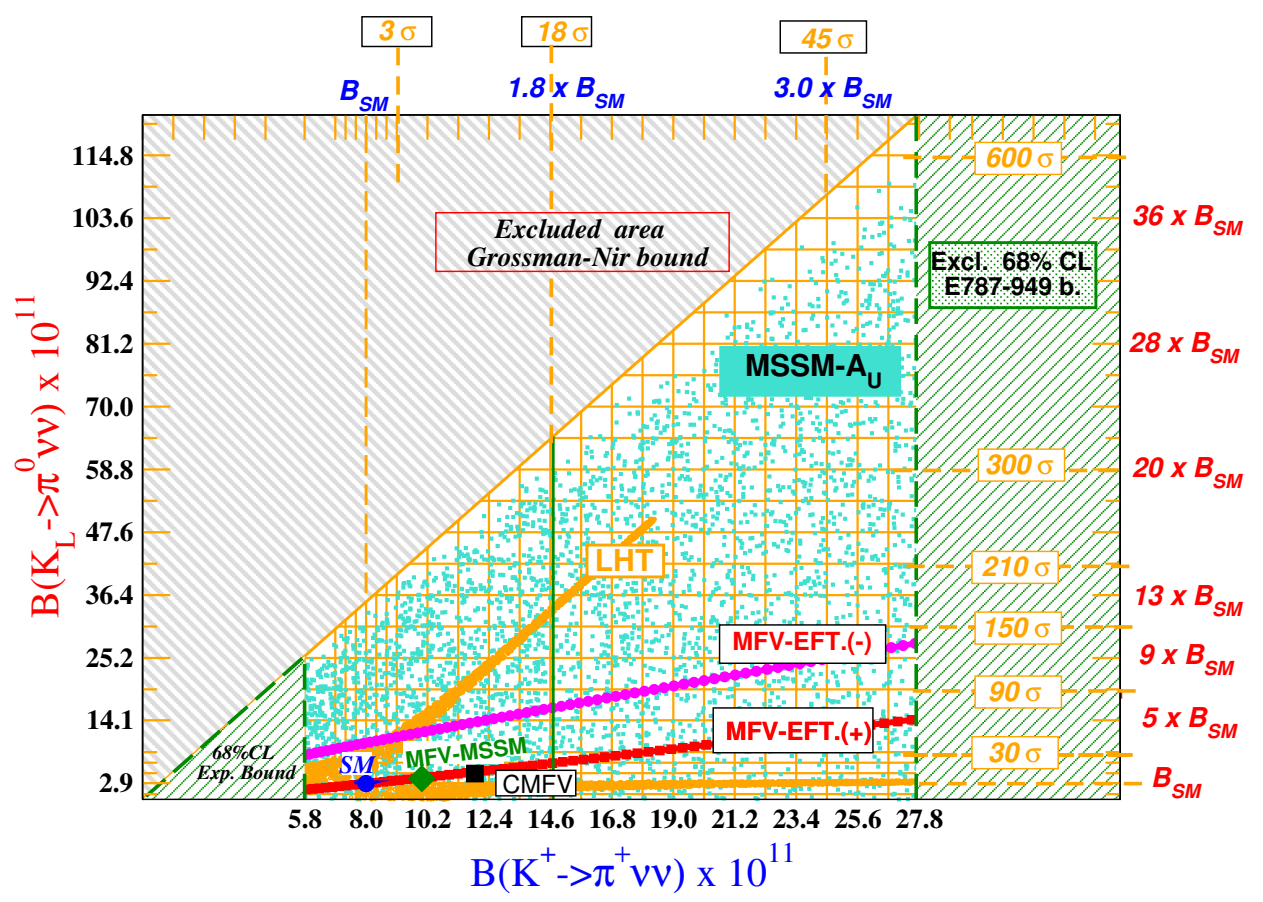

Figure 10: Predictions of different NP models for $\mathscr{B}\left(K^{+} \rightarrow \pi^{+} v \bar{v}\right)$ and $\mathscr{B}\left(K^{+} \rightarrow \pi^{+} v \bar{v}\right)$ [courtesy of F. Mescia]. The $95 \%$ C.L. exluded areas of $\mathscr{B}\left(K^{+} \rightarrow \pi^{+} v \bar{v}\right)$ refer to the result of the BNL-E787/949 experiment [58]

very promising prospects for the charged and the neutral channel, respectively. These challenging experiments, which aim at $\mathscr{O}(10 \%)$ measurements of the branching ratios, should receive the strongest support of our community. Their results will be very interesting also in the LHC era: they will alllow us to investigate the flavour structure of physics beyond the SM [61]. Needless to say that further proposals (or upgrades) would also be extremely welcome, since neither P326 nor the JPARC experiment will be able to fully exploit the discovery potential of these rare modes.

\section{Conclusions}

This conference has clearly demonstrated the high interest of the recent results obtained in kaon physics, and the promising future of this field. As I tried to outline, kaon physics is still a privileged observatory for improving the determination of fundamental SM parameters (such as $V_{u s}$ and $m_{u, d, s}$ ); performing high-precision tests of weak-current universality (with important implications on beyond-the-SM phenomenology); understanding in a deeper way the structure of the QCD vacuum; testing the basic assumptions of quantum field theory; shedding light on the flavour structure of physics beyond the SM.

We learned a lot from kaon physics, but exciting lessons could still be ahead of us ...

\section{Acknowledgment}

I wish to thank the organizers of this conference, and especially Caterina Bloise, for having created 
a very stimulating atmosphere. A special thank is due to Paolo Franzini, who proposed me for this summary: now that is over, I should admit that it has been a very instructive experience!

\section{References}

[1] M. Palutan, these proceedings.

[2] W. Marciano, these proceedings.

[3] T. Appelquist and J. Carazzone, Phys. Rev. D 11 (1975) 2856.

[4] J. Stern, these proceedings.

[5] P. Paradisi, these proceedings.

[6] D. Gorbunov, these proceedings.

[7] V. Cirigliano, these proceedings.

[8] All the averages reported in this review refer to results obtained by the Flavianet Kaon WG [http://www.lnf.infn.it/wg/vus/].

[9] W. M. Yao et al. [Particle Data Group], J. Phys. G 33 (2006) 1.

[10] B. Sciascia, these proceedings.

[11] P. Massarotti, these proceedings.

[12] A. Dabrowski, these proceedings.

[13] V. Duk, these proceedings.

[14] V. Cirigliano, H. Neufeld and H. Pichl, Eur. Phys. J. C 35 (2004) 53 [arXiv:hep-ph/0401173].

[15] P. Franzini , these proceedings; C. Gatti, these proceedings.

[16] H.Bijnens, these proceedings, arXiv:0707.0419 [hep-ph].

[17] T. Kaneko, these proceedings.

[18] A. Juettner, these proceedings.

[19] J. Bijnens and P. Talavera, Nucl. Phys. B 669 (2003) 341 [arXiv:hep-ph/0303103].

[20] D. J. Antonio et al., arXiv:hep-lat/0702026.

[21] V. Cirigliano et al. JHEP 0504 (2005) 006 [arXiv:hep-ph/0503108].

[22] C. Bernard et al., arXiv:hep-lat/0611024.

[23] A. Masiero, P. Paradisi, and R. Petronzio Phys. Rev. D 74, 011701 (2006) [arXiv:hep-ph/0511289].

[24] R. Wanke, these proceedings, arXiv:0707.2289 [hep-ex].

[25] L. Fiorini, PoS HEP2005, 288 (2006).

[26] V. Kozhuharov, these proceedings.

[27] A. Sibidanov, these proceedings.

[28] G. Ruggiero, these proceedings.

[29] G. Colangelo, J. Gasser and H. Leutwyler, Phys. Lett. B 488 (2000) 261 [hep-ph/0007112]; Nucl. Phys. B 603 (2001) 125. 
[30] G. Colangelo, these proceedings.

[31] J. Gasser, these proceedings.

[32] N. Cabibbo, Phys. Rev. Lett. 93 (2004) 121801 [hep-ph/0405001].

[33] L. Di Lella these proceedings; E. Goudzovski, these proceedings, arXiv:0706.4059 [hep-ex].

[34] N. Cabibbo and G. Isidori, JHEP 0503 (2005) 021 [hep-ph/0502130].

[35] G. Colangelo, J. Gasser, B. Kubis and A. Rusetsky, Phys. Lett. B 638 (2006) 187 [hep-ph/0604084].

[36] E. Gamiz, J. Prades and I. Scimemi, Eur. Phys. J. C 50 (2007) 405 [arXiv:hep-ph/0602023].

[37] S. Pislak et al., Phys. Rev. D 67 (2003) 072004 [arXiv:hep-ex/0301040].

[38] B. Bloch-Devaux, these proceedings.

[39] C. Sachrajda, these proceedings; R. Mawhinney, these proceedings.

[40] J. R. Batley et al. [NA48/2 Collaboration], arXiv:0707.0697 [hep-ex].

[41] G. D’Ambrosio, G. Isidori and N. Paver, Phys. Lett. B 273 (1991) 497.

[42] J. Prades, these proceedings, arXiv:0707.1789 [hep-ph].

[43] E. Gamiz, J. Prades and I. Scimemi, JHEP 0310 (2003) 042 [arXiv:hep-ph/0309172].

[44] G. D’Ambrosio, G. Isidori and G. Martinelli, Phys. Lett. B 480 (2000) 164 [arXiv:hep-ph/9911522].

[45] E. Cheu, these proceedings.

[46] M. Martini, these proceedings.

[47] G. Buchalla, G. D’Ambrosio and G. Isidori, Nucl. Phys. B 672 (2003) 387 [arXiv:hep-ph/0308008].

[48] N. E. Mavromatos, these proceedings, arXiv:0707.3422 [hep-ph].

[49] F. Ambrosino et al., JHEP 0612 (2006) 011 [arXiv:hep-ex/0610034].

[50] M. Testa, these proceedings.

[51] F. Ambrosino et al. [KLOE Collaboration], Phys. Lett. B 642 (2006) 315 [arXiv:hep-ex/0607027].

[52] U. Haisch, arXiv:0707.3098 [hep-ph].

[53] C. Tarantino, arXiv:0706.3436 [hep-ph].

[54] C. Smith, arXiv:0707.2309 [hep-ph].

[55] A. J. Buras, M. Gorbahn, U. Haisch and U. Nierste, Phys. Rev. Lett. 95, 261805 (2005) [arXiv:hep-ph/0508165]; JHEP 0611, 002 (2006) [arXiv:hep-ph/0603079].

[56] G. Isidori, F. Mescia and C. Smith, Nucl. Phys. B 718, 319 (2005) [arXiv:hep-ph/0503107].

[57] F. Mescia and C. Smith, Phys. Rev. D 76 (2007) 034017 [arXiv:0705.2025 [hep-ph]].

[58] V. V. Anisimovsky et al. [E949 Collaboration], Phys. Rev. Lett. 93 (2004) 031801 [arXiv:hep-ex/0403036].

[59] G. Perdue, these proceedings; T. Sumida these proceedings.

[60] H. Watanabe, these proceedings.

[61] A. J. Buras et al. in Flavour in the era of the LHC, CERN Yellow Report, to appear; G. Isidori, [arXiv:hep-ph/0606047]. 